\title{
Evaluation of the diagnostic value of the tourniquet test in predicting severe dengue cases in a population from Belo Horizonte, State of Minas Gerais, Brazil
}

\author{
Alice Costa Antunes ${ }^{[1]}$, Geancarlo Laporte Oliveira ${ }^{[1]}$, Leidiane Ileila Nunes ${ }^{[1]}$, \\ Luiz Alberto Guedes Filho ${ }^{[1]}$, Rafael Santos Prado ${ }^{[1]}$, Hugo Rezende Henriques ${ }^{[2]}$ \\ and Aristides José Carvalho Vieira ${ }^{[3]}$
}

[1]. Curso de Medicina, Faculdade da Saúde e Ecologia Humana, Vespasiano, MG. [2]. Departamento de Parasitologia, Faculdade de Medicina de Ribeirão Preto, Universidade de São Paulo, Ribeirão Preto, SP. [3]. Departamento de Medicina Social e Preventiva, Faculdade da Saúde e Ecologia Humana, Vespasiano, MG.

\begin{abstract}
Introduction: Dengue is prevalent in many tropical and sub-tropical regions. The clinical diagnosis of dengue is still complex, and not much data are available. This work aimed at assessing the diagnostic accuracy of the tourniquet test in patients with suspected dengue infection and its positivity in different classifications of this disease as reported to the Information System for Notifiable Disease in Belo Horizonte, State of Minas Gerais, Brazil between 2001 and 2006. Methods: Cross-section analysis of the diagnostic accuracy of the tourniquet test for dengue, using IgM-anti-DENV ELISA as a gold standard. Results: We selected 9,836 suspected cases, of which $41.1 \%$ were confirmed to be dengue. Classic dengue was present in $95.8 \%$, dengue with complications in $2.5 \%$ and dengue hemorrhagic fever in $1.7 \%$. The tourniquet test was positive in $16.9 \%$ of classic dengue cases, $61.7 \%$ of dengue cases with complications and $82.9 \%$ of cases of dengue hemorrhagic fever. The sensitivity and specificity of the tourniquet test were $19.1 \%$ and $86.4 \%$, respectively. Conclusions: A positive tourniquet test can be a valuable tool to support diagnosis of dengue where laboratory tests are not available. However, the absence of a positive test should not be read as the absence of infection. In addition, the tourniquet test was demonstrated to be an indicator of dengue severity.
\end{abstract}

Keywords: Dengue. Tourniquet test. Diagnosis. Brazil.

\section{INTRODUCTION}

Dengue is an arboviral infection elicited by the dengue virus (DENV) and is present throughout most tropical and subtropical regions. Approximately 2.5 billion people live in endemic areas, and approximately 50 million dengue cases are reported annually ${ }^{1}$. In Belo Horizonte, State of Minas Gerais, Brazil, dengue is a major threat, causing both morbidity and mortality. The average incidence between 1996 and 2001 was 750 cases/100,000 people per year ${ }^{2}$.

Classifying the different outcomes of DENV infection is complicated, and different systems have been proposed, such as those from the World Health Organization (WHO) and the Brazilian Health Ministry (BHM). According to WHO guidelines from 1997, the most common system adopted worldwide, the clinical outcome of DENV infection should be classified into three main categories: 1) dengue fever (DF), 2) dengue hemorrhagic fever (DHF) and 3) dengue shock

Address to: Dr. Hugo Rezende Henriques. Depto Parasitologia/FMRP/USP. Campus USP. Av. Bandeirantes 3900, Monte Alegre, 14040-906 Ribeirão Preto, SP, Brasil.

Phone: 55 16 9-8114-2414

e-mail: hugorezende20@usp.br

Received 19 August 2013

Accepted 8 October 2013 syndrome ${ }^{3}$. However, the 2011 BHM guidelines classify the clinical manifestations of DENV infection into four groups: 1) classic dengue, 2) complicated dengue, 3) DHF and 4) dengue shock syndrome ${ }^{4}$ (Table 1).

Virus isolation (Riman's Test) is the gold standard for confirmation of DENV infection ${ }^{1}$. However, because of its complexity and cost, the daily use of this method in diagnosis is restricted ${ }^{5}$. The ELISA anti-IgM-DENV-specific assay also has a high degree of reliability (an average of $98 \%$ specificity and $90 \%$ sensitivity ${ }^{6}$, and as this method is both quicker and cheaper, it is generally more suitable for large-scale usage. The ELISA assay is used for comparative analysis in many international studies ${ }^{7}$; because it has high sensitivity and does not require much training to perform ${ }^{8}$, it is the routinely used test for detection of both IgG and IgM. However, fast-track diagnostic kits for dengue infections, although available, are not as specific or sensitive as advertised by manufacturers 9 .

Despite the large number of available tests for diagnosing dengue, many endemic areas cannot afford them, resulting in the need for the development of easy and inexpensive methods to guide clinical diagnosis of dengue ${ }^{7}$. Such is the case of the tourniquet test (TT), indicated by the WHO in 2011 as one of the diagnostic criteria for dengue, in spite of the different clinical outcomes $^{1}$. In Brazil, the TT is mandatory in all suspected cases of dengue and can be used as a possible criterion for diagnosing DHF, although never as the sole criterion for such classification ${ }^{4}$. 
TABLE 1 - Comparison of dengue clinical classifications, adapted from different guidelines.

\begin{tabular}{cc}
\hline Brazilian Health Ministry (2011) & World Health Organization (1997) \\
Classic dengue &
\end{tabular}

(Fever for 7 days at most, along with at least 2 of the following: headache, retroorbital pain, muscle pain, joint pain, rash and fatigue)

Complicated dengue

(Cases that do not suit the dengue hemorrhagic fever criteria and for which the classic dengue classification is unsatisfactory)
Dengue fever

(Fever along with at least 2 of the following: headache, retroorbital pain, muscle pain, joint pain and rash. May be accompanied by bleeding complications such as epistaxis, gingival bleeding, gastrointestinal bleeding, hematuria)
Dengue hemorrhagic fever

(Increased vascular permeability, thrombocytopenia and hemorrhagic manifestations)
Dengue hemorrhagic fever

(Dengue fever with hemorrhagic manifestations such as petechiae and mucosal or gastro-intestinal bleeding)

$$
\text { Dengue shock syndrome }
$$

(Severe cases of dengue hemorrhagic fever with critical plasma leakage leading to hypovolemic shock)

\section{Dengue shock syndrome}

(Severe cases of dengue hemorrhagic fever with critical plasma leakage leading to hypovolemic shock)
Positivity for the TT indicates capillary fragility and propensity to hemorrhagic events in the patient ${ }^{10}$. However, the result might be negative or weakly positive in patients in profound shock, turning positive, and even strongly so, right after recovery from shock ${ }^{3}$.

Previous data from a variety of studies have evaluated the sensitivity of the TT in detecting DHF, but the conclusions ranged from $0 \%{ }^{11}$ to $57 \%{ }^{12}$. A couple of other studies from 2000 and 2004 demonstrated the difficulty of using the TT alone to differentiate DHF (27\% and $47 \%$ positives, respectively) and DF (26\% and $39 \%$ positives, respectively) $)^{13,14}$. Moreover, these studies also reported that the TT was positive in cases of nondengue-related fever in $12 \%{ }^{14}$ and $5 \%{ }^{13}$ of cases.

A study conducted in Bangkok, Thailand reported low capacity for the TT in distinguishing the different clinical outcomes of DENV infection: $88 \%$ positive in cases of dengue shock syndrome, $94 \%$ positive in cases of $\mathrm{DHF}$ and $90 \%$ positive in cases of DF.

To date, no study has been completed to evaluate the use of the TT in endemic areas of Brazil. In this study, we analyzed sensitivity, specificity and positive and negative predictive values for the TT in the clinical diagnosis of DENV infections and its positivity in the different classifications of the disease in a database of patients registered with suspected dengue cases in the Sistema de Informação de Agravo de Notificação (SINAN) in Belo Horizonte, State of Minas Gerais, Brazil, between 2001 and 2006.

\section{METHODS}

The study was conducted using data available from the SINAN database, where files from patients from Belo Horizonte with suspected dengue cases were analyzed. The database is accessible to all health professionals and to the community in general. The study was approved by the Research Ethics Committee of the Faculdade da Saúde e Ecologia Humana. Informed consent was not required from the patients because the database does not display patients' personal information.

\section{Subjects}

We analyzed 25,673 suspected dengue case reports between 2001 and 2006 and selected all of those cases (9,836 reports) that met the following criteria: 1) suspected dengue cases in which the enzyme-linked immunosorbent assay-immunoglobulin $\mathrm{M}$ (ELISA-IgM) test was conducted and 2) suspected dengue cases in which the tourniquet test was conducted and the form was correctly completed (either positive or negative). No cases of dengue shock syndrome were reported during this period.

\section{Tourniquet test}

The tourniquet test was conducted according to the BHM guidelines based on the WHO guidelines as follows: the TT was performed by inflating a blood pressure cuff to a pressure midway between the systolic and diastolic pressures for 5 minutes. The test was considered positive when 20 or more petechiae per sq. inch were observed in adults and 10 or more petechiae per sq. inch in children ${ }^{44,17}$.

\section{Statistical analysis}

The data were analyzed using Microsoft Excel 2010. Specificity, sensitivity, positive and negative predictive values and the accuracy of the TT were all calculated based on the ELISA IgM-anti-DENV test, our gold standard ${ }^{18}$. The data were compared using the chi-squared test or the $\mathrm{Z}$ test for proportions. 


\section{RESULTS}

We selected 9,836 cases of suspected dengue infection that were analyzed using ELISA IgM-anti-DENV. A total of 4,047 cases were serum-positive $(41.1 \%$; $95 \%$ CI $40.2-42.1 \%)$ and were further classified according to clinical outcomes into 3,878 (95.8\%) cases of classic dengue, 99 (2.6\%) cases of complicated dengue and $70(1.8 \%)$ cases of DHF.

\section{Tourniquet test diagnostic performance}

As previously stated, we defined the ELISA IgM-anti-DENV as our gold standard for this study and evaluated the diagnostic parameters of the TT in the clinical detection of dengue infection. The analysis identified 774 dengue-infected patients as TT-positive, 789 non-infected patients as TT-positive, 3,273 dengue-infected patients as TT-negative and 5,000 non-infected patients as TT-negative.

From these data, we were able to calculate the sensitivity (19.1\%), specificity $(86.4 \%)$, positive predictive value (49.5\%), negative predictive value $(60.4 \%)$, accuracy $(58.7 \%$ or 0.59$)$, positive likelihood value (1.40) and negative likelihood ratio (0.94) (Table 2 and Table 3).

TABLE 2 - Number of positive and negative reported cases for the tourniquet test and ELISA IgM.

\begin{tabular}{lcc}
\hline & \multicolumn{2}{c}{ ELISA IgM } \\
\cline { 2 - 3 } & positive & negative \\
\hline Tourniquet test & 774 & 789 \\
Positive & 3,273 & 5,000 \\
Negative & \\
\hline
\end{tabular}

ELISA: enzyme-linked immunosorbent assay IgM: immunoglobulin M.

TABLE 3 - Tourniquet test diagnostic parameters in confirmed dengue cases in Belo Horizonte between 2001 and 2006.

\begin{tabular}{lc}
\hline Tourniquet test & Percentage \\
\hline Sensitivity & 19.1 \\
Specificity & 86.4 \\
False positive & 13.6 \\
False negative & 80.9 \\
Positive predictive value & 49.5 \\
Negative predictive value & 60.4 \\
Accuracy & 0.6 \\
Positive likelihood ratio & 1.4 \\
Negative likelihood ratio & 0.9 \\
\hline
\end{tabular}

\section{Tourniquet test positivity according to clinical outcomes}

Next, we calculated the positivity of the TT in the different clinical outcomes of dengue infection, according to the BHM 2011 guidelines - comparable to those available in the WHO 1997 guidelines, according to Table $\mathbf{1}$.

Analyzing all cases of suspected dengue infection, the rate of TT positivity was $15.9 \%$ (95\% CI 15.20-16.6\%). When we analyzed the positivity of the test only in the confirmed cases of dengue infection, the rate of TT positivity was $19.2 \%$ (95\% CI 17.90-20.3\%). Cases of suspected dengue infection that were excluded in serological analysis demonstrated a TT positivity of $13.7 \%$ (95\% CI $12.70-14.5 \%)$.

We then analyzed the positivity of each of the different clinical outcomes. As previously stated, no cases of dengue shock syndrome were reported in Belo Horizonte during the time period we researched. The rates of TT positivity were $16.9 \%(95 \% \mathrm{CI}$ 15.70-18.1\%) for classic dengue, $61.7 \%$ (95\% CI 52.00-71.2\%) for complicated dengue and $82.9 \%$ (95\% CI 74.00-91.7\%) for DHF.

We used statistical analysis to compare the positivity of the three groups of different clinical outcomes: by the chi-squared test, all three groups exhibited significant differences ( $\mathrm{p}$-value $<0.01$ ). We also compared each couple of groups separately by means of the $Z$ test for proportions: significant differences were noted between any of the analyzed couples - classic dengue vs. complicated dengue ( $p$-value $<0.01$ ), classic dengue vs. DHF ( $p$-value $<0.01$ ) and complicated dengue vs. DHF ( $p$-value $<0.01$ ). Thus, we can conclude that the TT provides significantly distinct positivity values to each of the dengue clinical outcomes adopted by the Brazilian Government (Table 4).

We also analyzed positivity of the TT for DF as classified in the WHO guidelines from 1997. That classification includes both classic dengue and complicated dengue; thus, we grouped these two categories together for this analysis. The positivity of the tourniquet test was 18\% (95\% CI 16.80-19.2\%) (Table 5).
TABLE 4 - Differences in tourniquet test positivity between groups according to the Z-test for proportions.

\begin{tabular}{|c|c|}
\hline & p-value \\
\hline \multicolumn{2}{|l|}{ Classic dengue positivity $16.9 \%(15.7-18.1 \%)$} \\
\hline versus & p-value $<0.01$ \\
\hline \multicolumn{2}{|l|}{ Complicated dengue positivity $61.7 \%(52.0 \%-71.2 \%)$} \\
\hline \multicolumn{2}{|l|}{ Classic dengue positivity $16.9 \%(15.7-18.1 \%)$} \\
\hline versus & p-value $<0.01$ \\
\hline \multicolumn{2}{|l|}{ Dengue hemorrhagic fever $82.9 \%$ (74.0\%-91.7\%) } \\
\hline \multicolumn{2}{|l|}{ Complicated dengue positivity $61.7 \%(52.0 \%-71.2 \%)$} \\
\hline versus & p-value $<0.01$ \\
\hline Dengue hemorrhagic fever $82.9 \%$ (74.0\%-91.7\%) & \\
\hline
\end{tabular}


TABLE 5 - Tourniquet test positivity.

\begin{tabular}{lr}
\hline Dengue cases & TT positivity \% (95\%CI) \\
\hline Suspected dengue cases & $15.9(15.2-16.6 \%)$ \\
Confirmed cases of dengue & $19.2(17.9-20.3 \%)$ \\
Non-dengue cases & $13.7(12.7-14.5 \%)$ \\
Classic dengue (BHM 2011) & $16.9(15.7-18.1 \%)^{*}$ \\
Complicated dengue (BHM 2011) & $61.7(52.0-71.2 \%)^{*}$ \\
Dengue hemorrhagic fever (BHM 2011. WHO 1997) & $82.9(74.0-91.7 \%)^{*}$ \\
Dengue fever (WHO 1997) & $18.0(16.8-19.2 \%)$ \\
\hline
\end{tabular}

*Groups significantly different. BHM: Brazilian Health Ministry; WHO: World Health Organization; 95\%CI: 95\% confidence interval; TT: tourniquet test.

\section{DISCUSSION}

In this work, we address the clinical diagnosis of dengue, a common problem faced by physicians in endemic areas, mainly due to the lack of proper diagnostic tools. It is problematic that only $41.1 \%$ of the 9,836 reported suspected dengue cases were confirmed to be positive serologically through laboratory analysis. Alone, these data should reveal the relevance of studies such as ours, which seeks to evaluate the actual performance of the TT, an inexpensive and simple clinical diagnostic test, so that we can enhance the accuracy and usefulness of the clinical diagnosis of this infection.

We analyzed all reported cases of suspected dengue in Belo Horizonte between 2001 and 2006. After excluding those cases not meeting our criteria, we were able to calculate the specificity and sensitivity of the TT. Our findings indicate that the TT has a high specificity but a low sensitivity, which means that although positive results for the TT are a strong indicator of dengue, negative tests cannot exclude the possibility of DENV infection. Similar studies have evaluated TT performance in different groups and places and have had similar results. A study in Vietnamese children reported a specificity as high as $94 \%$ but a sensitivity of only $42 \%$ for the TT in diagnosing suspected dengue infection ${ }^{19}$. Another study, this time evaluating suspected dengue infection in adults in Laos, also reported high (84\%) specificity and low (34\%) sensitivity 7 . Both studies had the ELISA assay as the gold-standard.

A previous study conducted in Malaysia tested children with suspected dengue infection daily using the TT and reported very different results: a low (23.5\%) specificity and a high (83\%) sensitivity. However, the author himself raised the possibility that such low specificity could be due to weakening of capillary strength, which results from repeatedly testing the same subject with the $\mathrm{TT}^{20}$.

However, our study differs from these previous works in that we analyzed a large amount of data, which bestows great statistical strength to our findings that, in general, corroborates most other studies in this field.
It is of particular interest that the difference in positivity of the TT in patients with confirmed dengue infection (19.2\%) and in patients with negative serology (13.6\%) is very narrow. Such a finding indicates the complexity of the clinical diagnosis of dengue infection and brings to light the main importance that health professionals should use a variety of clinical evidence to diagnose this infection correctly and differentiate it from other similar clinical conditions.

In spite of the fact that the TT positivity is similar between subjects with confirmed dengue infection and uninfected subjects, our other findings are very interesting. The TT presented higher positivity in cases of dengue infection with more severe outcomes (i.e., complicated dengue and DHF). Upon further analysis of this data, we can suppose that the TT can and should be used as a quick test to screen for the more severe outcomes of dengue infection and for patients who should be watched more closely.

The most recent WHO guidelines for prevention and control of dengue list the TT as one of the possible diagnostic criteria for dengue infection in general ${ }^{1,3}$. In these guidelines, there is strong concern for mistaken diagnosis of DF as DHF and viceversa $^{7}$. However, it is of critical importance to understand that, although the TT is an important tool for aiding in the clinical diagnosis of dengue infection and also highlights cases that most likely could develop into more severe clinical outcomes, the TT cannot and should not be used alone in diagnosing and classifying dengue infection.

A variety of studies, including ours, reported that DF patients could present with positive TTs, supporting the most recent WHO guidelines that highlight the value of the TT in indicating both DF and DHF. A previous study in Laos found that $33 \%$ of DF-confirmed patients where TT-positive and that $64 \%$ of DHF-confirmed patients where TT-positive 7 . Our data found that $18 \%$ and $83 \%$ of DF and DHF patients, respectively, presented with positive TTs. We believe that these findings indicate that the TT should only be used as evidence of dengue infection rather than as a conclusive diagnosis or a solo criterion for the classification of dengue clinical outcomes. Therefore, complimentary analysis must always be performed to diagnose 
and classify DENV infection correctly. That said, it is also important that we highlight that a positive TT should lead to a more careful look at the patient, as it seems to be a reliable indicator for the development of the more severe outcomes of dengue infection.

Our study allows us to conclude that a positive TT can be useful in supporting a dengue infection diagnosis, especially in regions where laboratory tests are not readily available options for confirmation. However, we also conclude that a negative TT should not be interpreted as evidence of non-infection.

Furthermore, we conclude that the TT is a good predictive tool that allows agents to foresee the most severe clinical outcomes of dengue infection and that a positive test result should be used to indicate those patients that should receive careful treatment from health agents.

\section{CONFLICT OF INTEREST}

The authors declare that there is no conflict of interest.

\section{REFERENCES}

1. World Health Organization (WHO). Comprehensive Guidelines for Prevention and Control of Dengue and Dengue Haemorrhagic Fever. $1^{\text {st }}$ ed. Geneva: WHO; 2011.

2. Corrêa PRL, França E, Bogutchi TF. Aedes aegypti infestation and occurrence of dengue in the City of Belo Horizonte, Brazil. Rev Saude Publica 2005; 39:33-40.

3. World Health Organization (WHO). Dengue haemorrhagic fever: diagnosis, treatment, prevention and control. $2^{\text {nd }}$ ed. Geneva: WHO; 1997.

4. Ministério da Saúde. Dengue: Diagnóstico e Manejo Clínico. $4^{\text {th }}$ ed. Brasília: Ministério da Saúde; 2011.

5. Rothman AL, Srikiatkhachorn A, Kalayanarooj S. Clinical presentation and diagnosis of dengue virus infections [Internet]. [Cited 2012 September 17] Available at: http://www.uptodate.com/contents/clinical-presentationand-diagnosis-of-dengue-virus-infections/. UpToDate; 2012.

6. Guzman MG, Kouri G, Gustavo K. Dengue diagnosis, advances and challenges. J Infect 2004; 8:69-80.
7. Mayxay M, Phetsouvanh R, Moore CE, Chansamouth V, Vongsouvath M, Sisouphone S, et al. Predictive diagnostic value of the tourniquet test for the diagnosis of dengue infection in adults. Trop Med Int Health 2011; 16:127-133.

8. Silva AM. Estudo de cinética de viremia do vírus dengue sorotipo $3 \mathrm{em}$ formas clínicas da Dengue com diferentes níveis de gravidade. Recife: Fundação Oswaldo Cruz; 2008.

9. Blacksell SD, Bell D, Kelley J, Mammen Jr MP, Gibbons RV, Jarman $R G$, et al. Prospective study to determine accuracy of rapid serological assays for diagnosis of acute dengue vírus infection in Laos. Clin Vaccine Immunol 2007; 14:1458-1464.

10. Bandyopadhyay S, Lum LC, Kroeger A. Classifying dengue: a review of the difficulties in using the WHO case classification for dengue haemorrhagic fever. Trop Med Int Health 2006; 11:1238-1255.

11. Pushpa V, Venkatadesikalu M, Mohan S, Cherian T, John TJ, Ponnuraj EM. An epidemic of dengue haemorrhagic fever/dengue shock syndrome in tropical India. Ann Trop Paediatr 1998; 18:289-293.

12. Kalayanarooj S, Vaughn DW, Nimmannitya S, Green S, Suntayakorn S, Kunentrasai N, et al. Early clinical and laboratory indicators of acute dengue illness. J Infect Dis 1997; 176: 313-321.

13. Phuong CX, Nhan NT, Kneen R, Thuy PT, van Thien C, Nga NT, et al. Clinical diagnosis and assessment of severity of confirmed dengue infections in Vietnamese children: is the world health organisation classification system helpful? Am J Trop Med Hyg 2004; 70:172-179.

14. Lucas GN, Amerasinghe A, Sriranganathan S. Dengue haemorrhagic fever in Sri Lanka. Indian J Pediatrics 2000; 67:503-504.

15. Kalayanarooj S, Chansiriwong V, Nimmannitya S. Dengue patients at the children's hospital, Bangkok: 1995-1999 review. Dengue Bulletin 2002; 26:3-43.

16. Instituto Brasileiro de Geografia e Estatística (IBGE). Censo 2010. Rio de Janeiro: IBGE; 2010. [Cited 2012 September 17]. Available at: http:// www.ibge.gov.br/home/presidencia/noticias/noticia_visualiza.php?id_ noticia $=1766 \&$. id_pagina $=1 /$

17. World Health Organization (WHO). Dengue: Guidelines for Diagnosis, Treatment, Prevention and Control. Geneva: WHO; 2009.

18. Altman DG. Practical statistics for medical research. Chapman And Hall, London; 1991.

19. Phuong CX, Nhan NT, Wills B, Kneen R, Nguyen TT, Mai TT, et al. Evaluation of the world health organization standard tourniquet test and a modifield tourniquet test in the diagnosis of dengue infection in Vietnam. Trop Med Int Health 2002; 7:125-132.

20. Norlijah O, Khamisah AN, Kamarul A, Paeds M, Mangalam S. Repeated tourniquet testing as a diagnostic tool in dengue infection. Med J Malaysia $2006 ; 61: 22-27$. 